To be Published in Ultra-Wideband, Short-Pulse Electromagnetics 3,

Proceedings of the $3^{\text {rd }}$ Int'l Conference on Ultra-Wideband, Short-Pulse Electromagnetics

(May 27-31, 1996, Albuquerque, NM)

$$
\begin{aligned}
& \text { SANO95.2383C } \\
& \text { CONF. } 9605185--3
\end{aligned}
$$

\title{
MEASUREMENTS OF SHORT-PULSE PROPAGATION THROUGH CONCRETE WALLS
}

\author{
Dr. John F. Aurand \\ Sandia National Laboratories ${ }^{1}$ \\ High-Power Electromagnetics Department 9323 \\ P.O. Box 5800, MS-1153 \\ Albuquerque, NM 87185-1153
}

\section{INTRODUCTION}

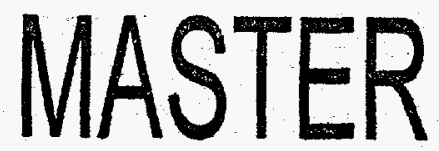

We recently performed a series of experimental measurements of transient electromagnetic (EM) propagation through two different concrete walls. Several different short-duration pulses were used for the incident radiation, with frequency content from VHF to $20 \mathrm{GHz}$. Both walls were $30 \mathrm{~cm}$ thick, with three internal layers of reinforcing steel bars. For this particular set of data, the incident wave polarization was vertical linear only. Corroborating swept-frequency measurements were made with a vector network analyzer.

This paper describes the propagation measurements through the two walls, and the propagation model of a lossy dielectric layer. We also examine the transfer function, dielectric constant, loss tangent, attenuation constant, and time-domain impulse response of these walls. The attenuation increases steadily with frequency, and is a strong function of the moisture content of the concrete. The time-domain pulse attenuation and dispersion are consistent with the lowpass-filtering effect of this attenuation loss vs. frequency.

\section{TIME-DOMATN PROPAGATION MEASUREMENTS}

The transmitter portion of the wideband time-domain measurement system consisted of a very fast low-voltage commercial pulse generator and a wideband TEM-horn antenna with dielectric aperture lens. The receiver consisted of an identical TEM-horn antenna and a wideband sampling oscilloscope. These new antennas are described more fully in the companion paper in this Proceedings, 'A TEM-Horn Antenna with Dielectric Lens for Fast Impulse Response,' and were expressly developed for this

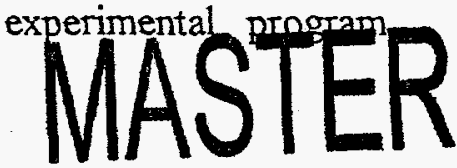

${ }^{1}$ This Work was Supported by the U.S. DOE under Contract DE-AC04-94AL85000. 
Considerable effort was spent in optimizing this measurement system to have the fastest transient response possible (thus offering the widest bandwidth of wall characterization).

In order to characterize the pulse attenuation and dispersion of a concrete wall, two different transmission measurements are made in order to form a transfer function representing the lossy dielectric layer under test. One is a free-space reference measurement, characterizing the measurement system response. The other is the 'through' measurement, in which the pulse is actually measured after propagating through the layer of interest. The transfer function is then given by the frequency-domain ratio of the processed received waveforms.

This paper presents data taken with two indoor concrete walls, at different facilities. One wall was located in one of our buildings at Sandia National Labs (Kirtland AFB, New Mexico), and the other wall was located in a building at Wright Laboratory/Armament Directorate (Eglin AFB, Florida). Figure 1 is a photograph of the Wright Lab experimental setup used to capture the measurement system response in a free-space boresight antenna alignment. The antenna aperture separation was $1.10 \mathrm{~m}$ (precisely the same as the separation for the wall measurement). The transmit antenna is pointed toward the viewer, with the pulse generator equipment located behind it; the dielectric lens can be seen in the aperture of the antenna. The receive antenna is pointed toward the transmit antenna, in this case with both main-beam boresight directions carefully aligned for maximum time-domain response. The receiving sampling oscillosope can be seen at the left side of the photo, sitting on top of a vector analyzer.

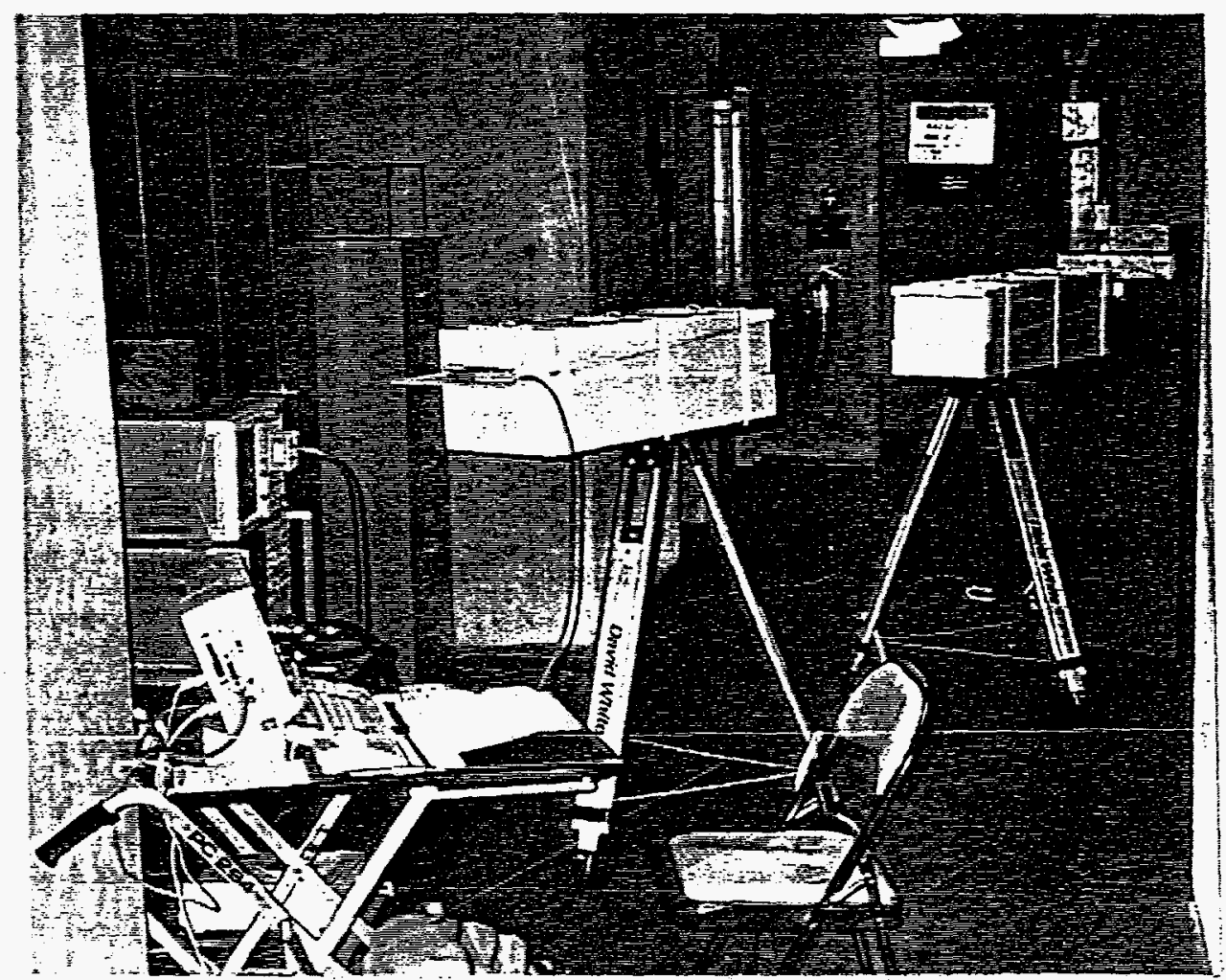

Figure 1. Free-space setup for reference measurement of system, at Wright Lab facility.

For the through measurements, Figures 2 and 3 show the transmitter and receiver setups, respectively. In Figure 2, the transmit antenna is oriented at normal incidence to the wall, with the aperture separated $40 \mathrm{~cm}$ from the front surface. In Figure 3, the receive antenna is seen in a similar orientation, on the opposite side of this wall. It is also easier to view the receive equipment in this figure. The antennas were very carefully positioned in 


\section{DISCLAMMER}

Portions of this document may be illegible in electronic image products. Images are produced from the best available original document. 

order to have a normally-incident propagation line-of-sight and repeatable wall separations (to an accuracy of better than $0.5 \mathrm{~cm}$ ).

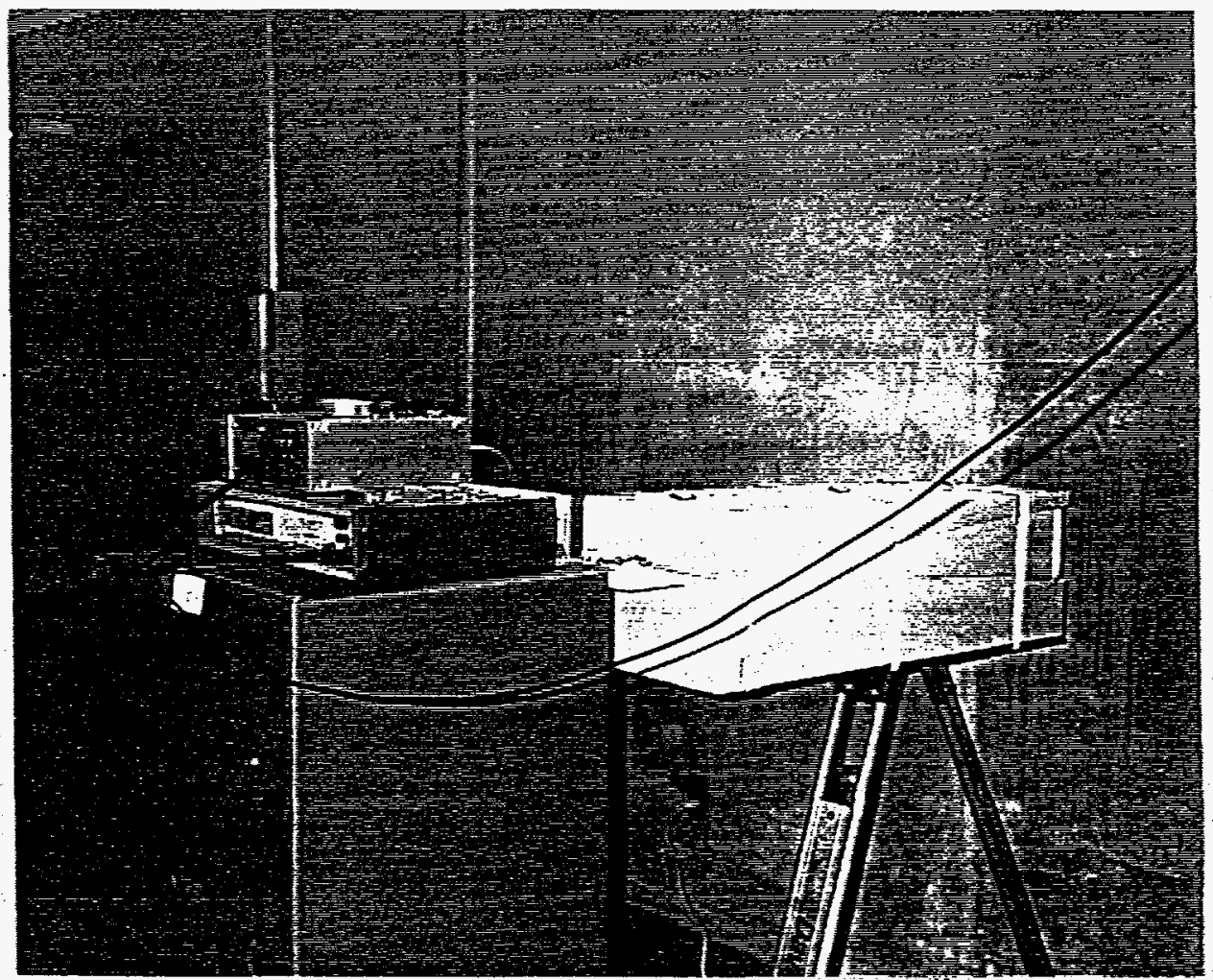

Figure 2. Transmitter setup for wall measurement, at Wright Lab facility.

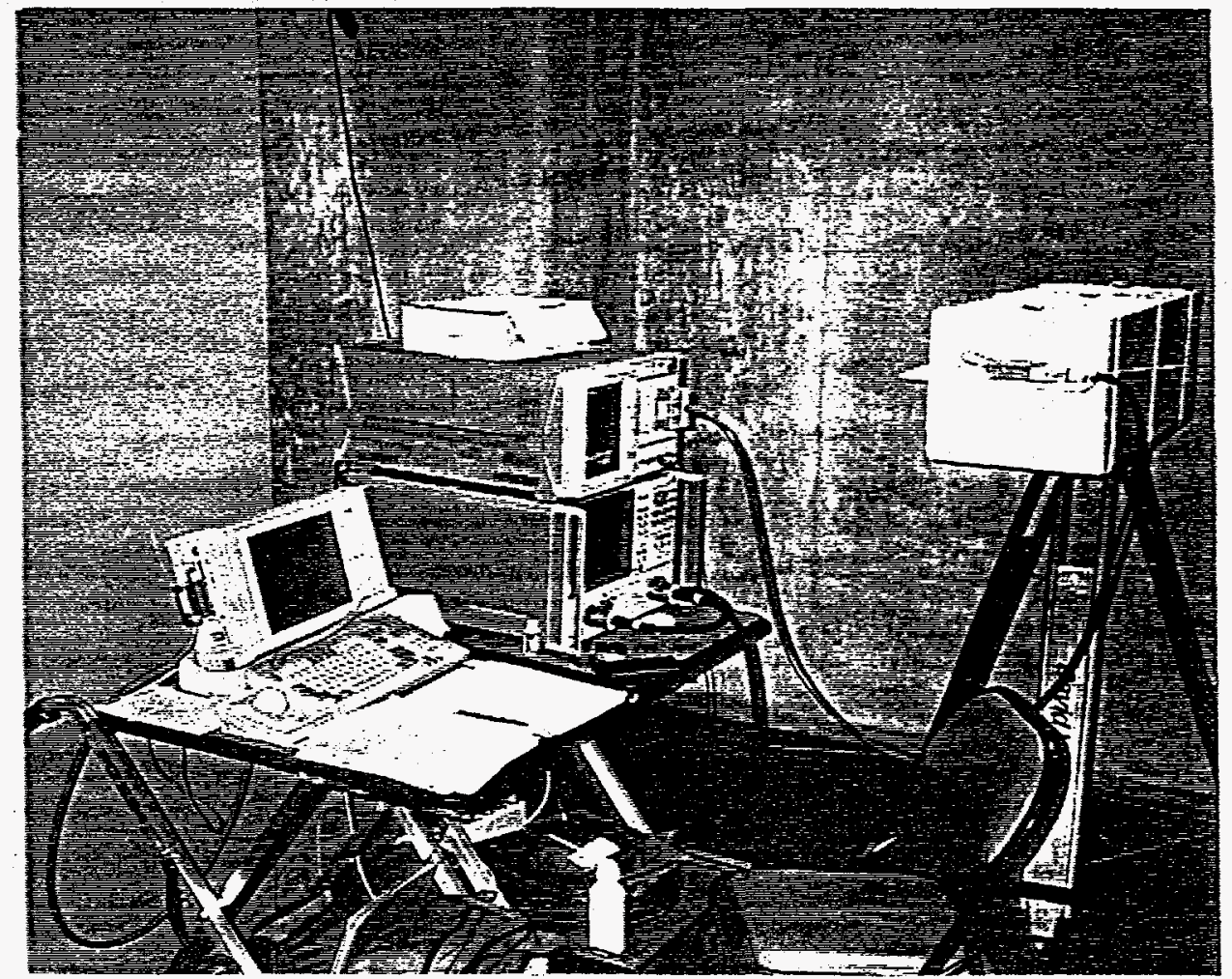

Figure 3. Receiver setup for wall measurement, at Wright Lab facility. 
The experimental setup at our facility consisted of the same equipment, but the antennas were supported by custom wood platforms rather than the wood tripods shown in the figures above. These platforms are shown in Figure 6 of the companion paper mentioned above. The aperture separation of the antennas was $90.5 \mathrm{~cm}$.

A variety of time-domain pulse types were used in these measurements in order to determine their transient propagation-loss behavior. These TEM-horn antennas have a clean derivative transmit time-domain response (on boresight), so the radiated EM field is a derivative of the pulse which drives the transmit antenna. The receive response is a time integral of the transmit response, so the antenina produces an output voltage pulse which is a replica of the incident wave. As a result, with step excitation of the transmit antenna, the radiated wave incident on the wall under test is an impulse, and the system has a free-space received pulse which is also an impulse. For the Sandia wall experiments, four impulse EM waves were created, with different pulse durations.

Figure 4 shows one set of data for the Sandia wall, using the fastest impulse excitation. The first and largest impulse is the free-space reference waveform, and the second waveform is that through the wall. The reference impulse has a $50 \%$ pulse duration (full-width at half-maximum) of $48 \mathrm{ps,} \mathrm{and} \mathrm{with} \mathrm{a} \mathrm{receiving} \mathrm{system} \mathrm{10-90 \%} \mathrm{transition}$ duration of about 22 ps (with a 50-GHz sampling head), this means that the radiated field incident on the wall has a pulse duration of about 43 ps. This is the fastest (and widest bandwidth) radiated-wave system we know of for measuring the transient microwave properties of layered dielectric materials. Also note the 1.3-ns time delay due to pulse retardation in the wall. Based on this additional delay, the dielectric constant (relative permittivity) of the wall is 5.2. A further item to note is the lowpass-filtering effect of the through measurement.

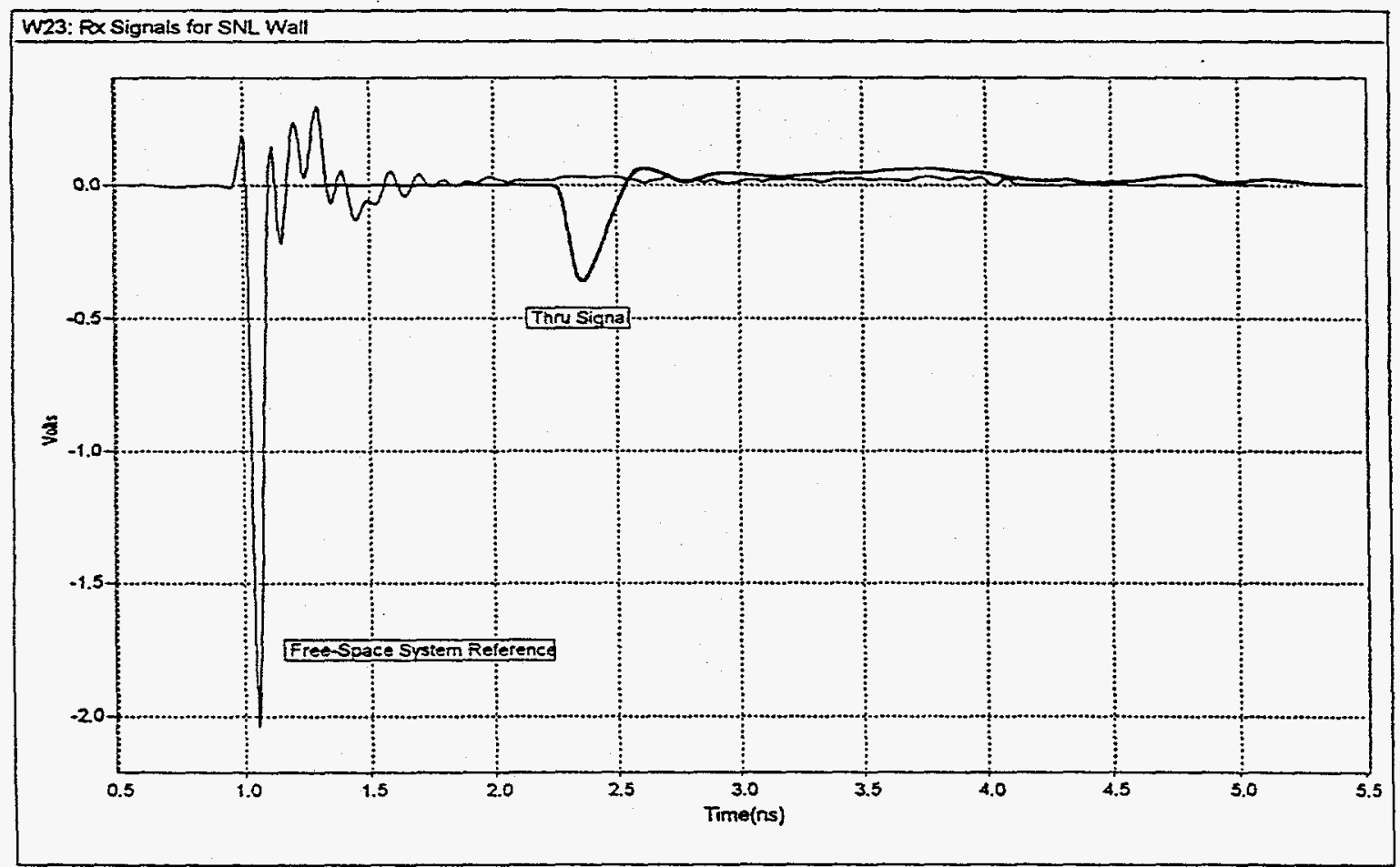

Figure 4. Received waveforms (free-space reference and through-wall), for Sandia Labs wall.

For the Wright Lab wall, five different impulse excitations were used, as well as a bipolar wave excitation. These data are shown in Figure 5, together with the corresponding through waveforms. Note the time delay through the wall $(1.15 \mathrm{~ns})$, and how consistent it is 
for the set of excitation waveforms. This implies that the dielectric constant is relatively constant over the spectral content of the excitation pulses (with a value of 4.5).

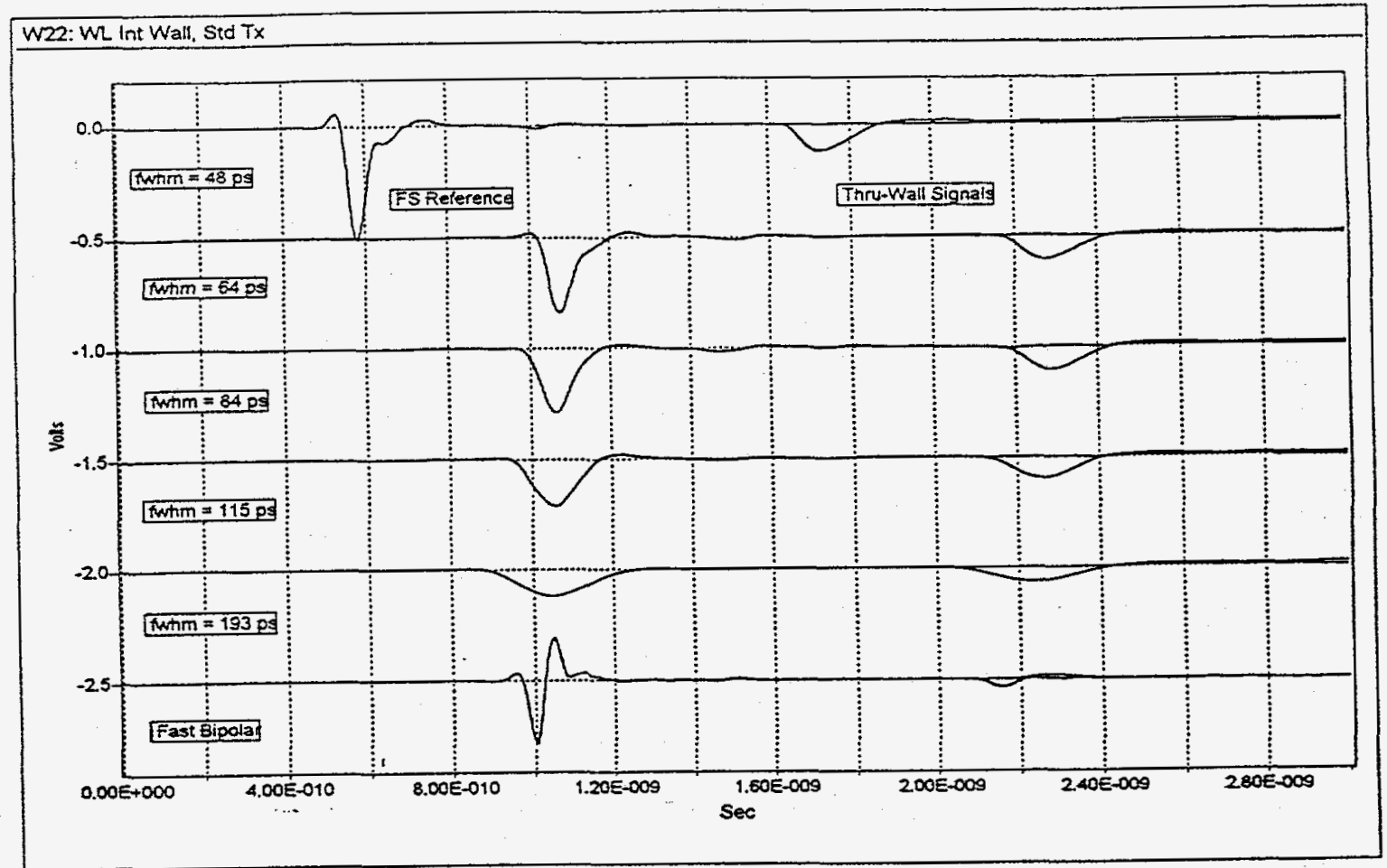

Figure 5. Received waveforms (free-space reference and through-wall), for Wright Lab wall. There are five different impulse excitations and one bipolar excitation.

\section{PROPAGATION MODEL \& DATA ANALYSIS}

In terms of characterizing the propagation through a wall or other lossy dielectric layer, the most straightforward configuration is a free-space transmission measurement, in which a radiated EM wave is launched by a transmitting antenna, propagates through a material layer of some thickness, and is captured by a receiving system. If we assume plane-wave propagation and a plane-wave layered geometry, then the problem becomes a 1-D model, and the material layer under test can be modeled as a two-port device, with an overall transfer function which relates the output pulse to the input excitation pulse. This transfer function can then be used to unfold or determine the dielectric constant and loss tangent of the material layer.

The single-bounce or partial reflection coefficient at the front surface of a lossy dielectric layer can be modeled as

$$
\Gamma(f) \equiv \frac{E_{r}(f)}{E_{i}(f)}=\frac{\eta-\eta_{0}}{\eta+\eta_{0}}=\frac{1-\sqrt{\varepsilon_{r}\left(1-j p_{e}\right)}}{1+\sqrt{\varepsilon_{r}\left(1-j p_{e}\right)}},
$$

where $E_{i}(f)$ and $E_{r}(f)$ are the Fourier spectra of the incident transient wave and the reflected wave from the front surface. The TEM-wave impedance in the layer is $\eta$, with a dielectric constant of $\varepsilon_{r}$ and an effective loss tangent of $p_{e}$. The corresponding complex propagation constant is

$$
\gamma(f) \equiv \alpha+j \beta=\frac{j \omega}{c} \sqrt{\varepsilon_{r}\left(1-j p_{e}\right)}
$$

and the resulting transfer function of the layer, based on a single-pass propagation pulse through it, is 


$$
H(f) \equiv \frac{E_{t}(f)}{E_{f s}(f)}=\left(1-\Gamma^{2}\right) e^{-\left(y-y_{0}\right) d}
$$

Here, $E_{t}(f)$ is the spectrum of the through pulse, and $E_{f_{s}}(f)$ is the spectrum of the free-space reference pulse. Since the same receiving system is used for both waveform measurements, this can also be obtained as the ratio of the received-voltage spectra rather than the wave fields themselves (i.e., it isn't necessary to deconvolve the receiving system out of the measurements).

Figure 6 shows the magnitude of this transfer function for the fastest impulse excitation of each of the walls, with a gradually increasing loss versus frequency. Note that the transfer functions based on all the other pulse excitations were very consistent with this data, but this fastest impulse excitation yielded the cleanest spectral characterization over the widest bandwidth (from UHF to $15 \mathrm{GHz}$ ).

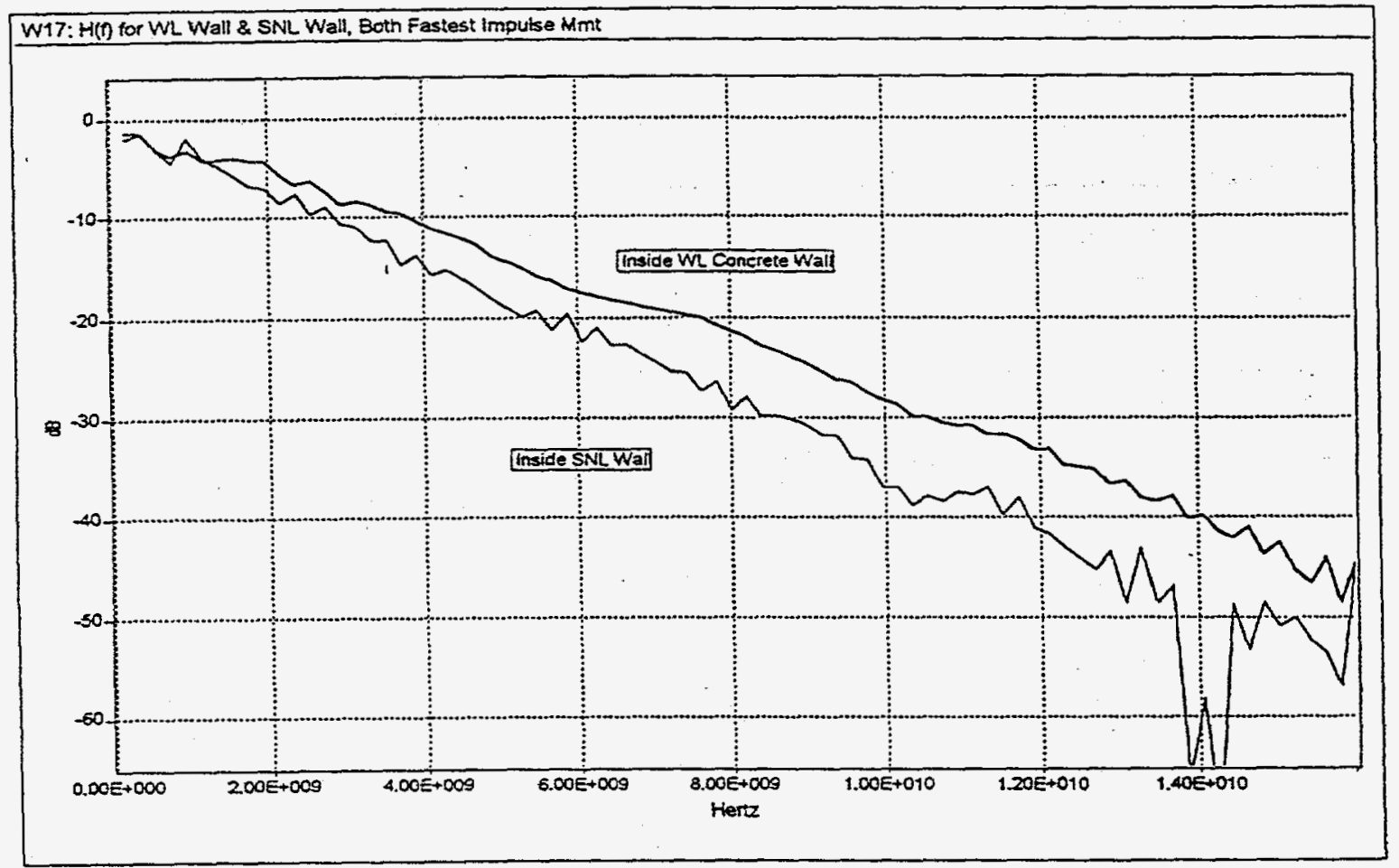

Figure 6. Transfer function for Sandia and Wright Labs' walls, based on fastest impulse excitations.

Then Figure 7 shows the corresponding impulse response of the walls, given by the inverse Fourier transform of the transfer function above. This is the pulse which would emerge from the concrete wall if excited with a perfect delta-function impulse TEM wave. There are several items to note about these waveforms. One is that the Wright wall has lower pulse attenuation and shorter time delay than the Sandia wall. The Wright wall has less attenuation and lower dielectric constant than the Sandia wall; this is evident in the frequency-domain transfer function as well. Note also that the primary impulse response is replica in nature, and that the average $50 \%$ pulse duration was 105 ps for the Wright wall and 146 ps for the Sandia wall. Thus, the Sandia wall has more time-domain dispersion or pulse spreading than the Wright wall. The last item to notice is the negative undershoot following the impulse portion of the response; we think that this is partially caused or influenced by the reinforcing bar inside the walls. 


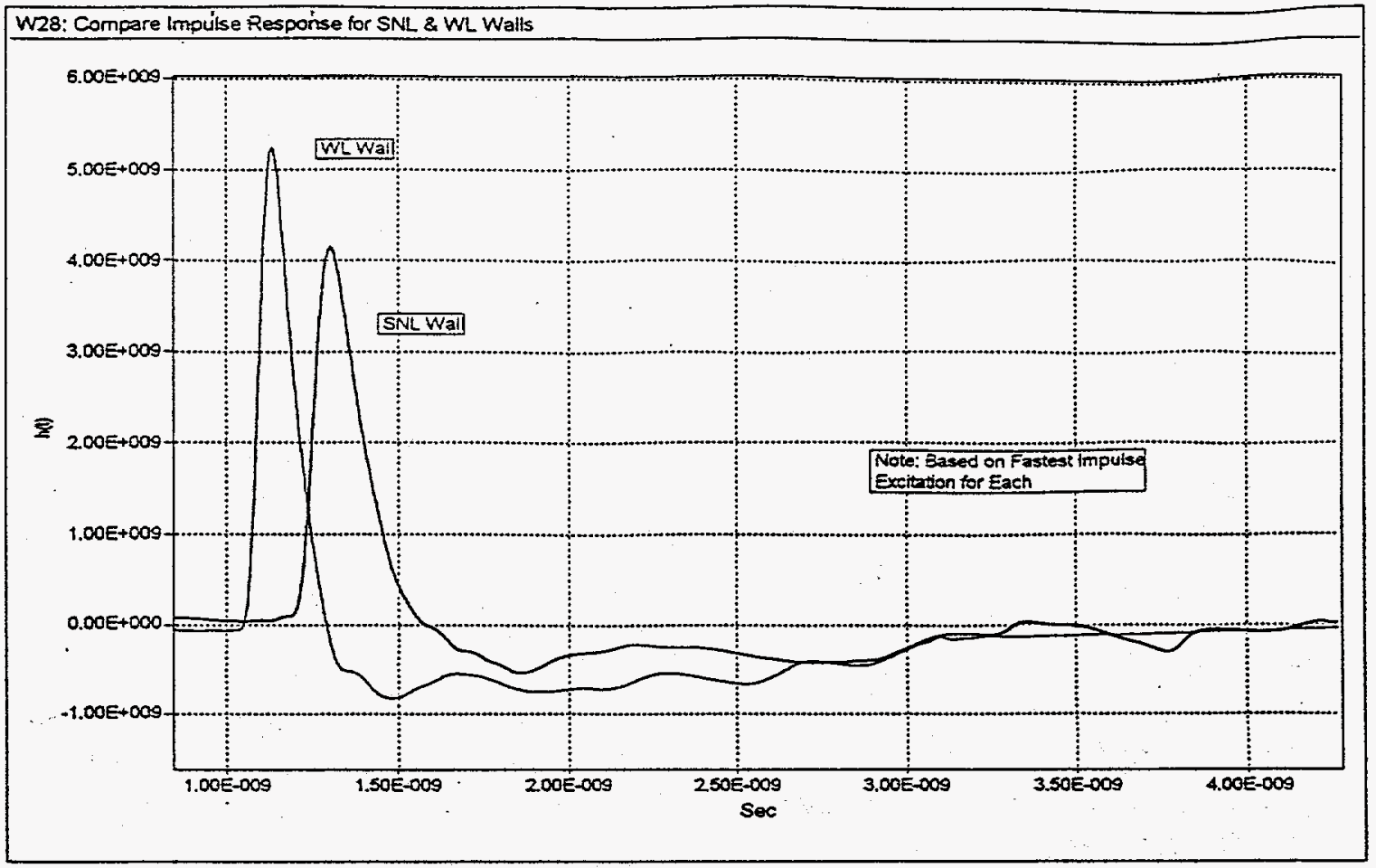

Figure 7. Impulse response of Sandia and Wright Labs' walls, based on inverse Fourier transform of transfer functions shown in Figure 6.

Space in this paper prohibits showing all of the data, but one more useful plot is provided in Figure 8 - this is the resulting attenuation constant $\alpha(f)$ in $\mathrm{dB} / \mathrm{m}$ for each wall. It represents the volumetric loss of the concrete and re-bar structure, with the pulse reflection losses at each layer interface having been removed.

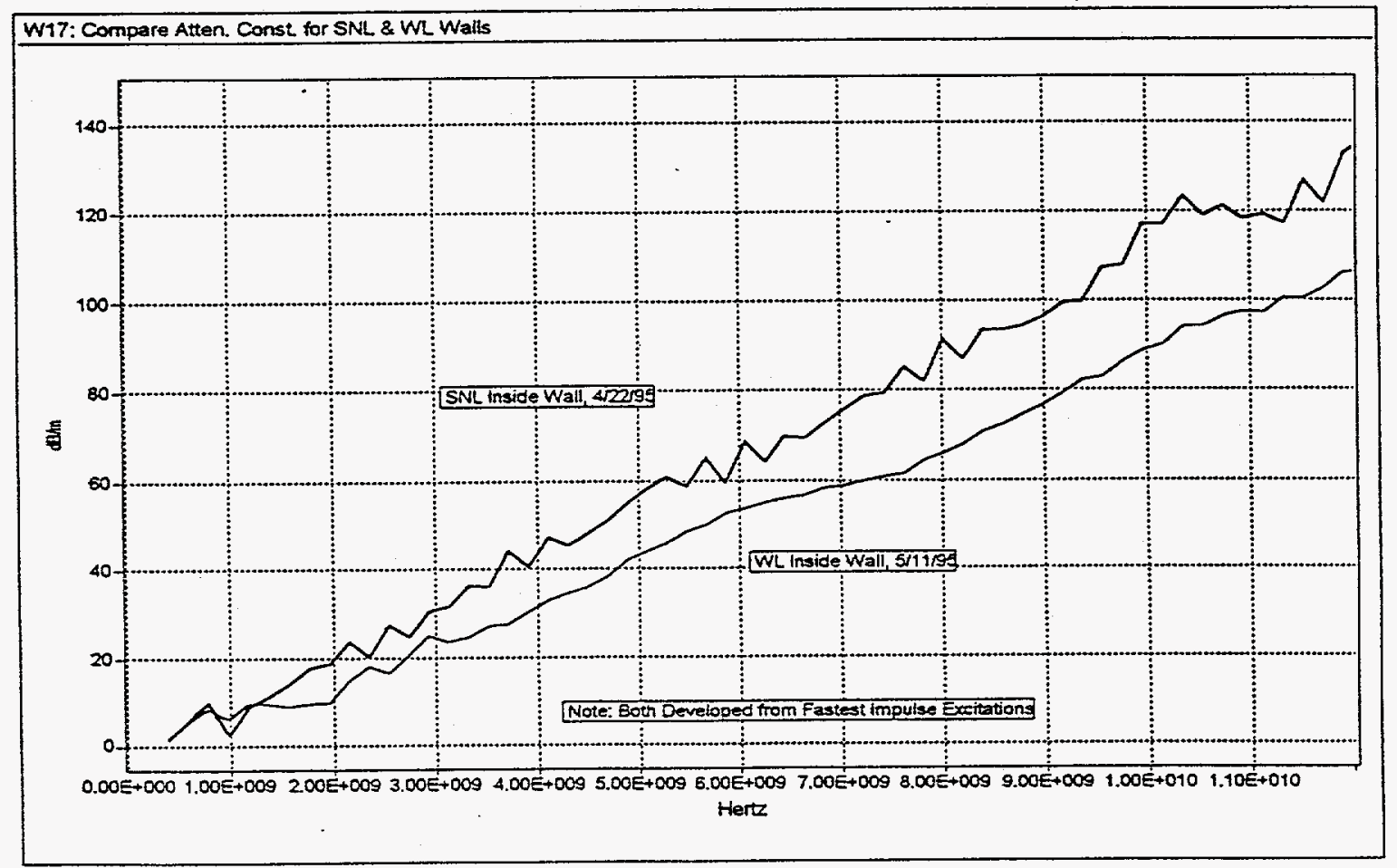

Figure 8. Attenuation constant of Sandia and Wright Labs' walls, based on the transfer functions shown in Figure 6. 
The Sandia wall does indeed have higher loss than the Wright wall, with about $20 \mathrm{~dB} / \mathrm{m}$ at

$2 \mathrm{GHz}$ and rising to about $110 \mathrm{~dB} / \mathrm{m}$ at $10 \mathrm{GHz}$. The corresponding dielectric constant is fairly flat over this range, consistent with simple time-delay behavior in the impulse response.

The time-domain cross-correlation of the waveform pairs shows similar behavior as the derived impulse response, with the same insertion delays and relative pulse attenuation.

\section{SUMMARY}

We performed two series of very careful propagation measurements through two different $30-\mathrm{cm}$-thick concrete walls, utilizing a variety of transient time-domain pulses. The resulting transfer function and corresponding time-domain impulse response were very consistent with each other, and were additionally validated by frequency-domain vector analyzer measurements of the transfer function. This is the first comprehensive data we have seen for very wideband characterization of actual concrete walls with re-bar construction. The attenuation constant (in $\mathrm{dB} / \mathrm{m}$ ) increases in a fairly linear fashion with frequency, and the dielectric constant is fairly flat over the UHF to $15-\mathrm{GHz}$ band. In addition, the time-domain behavior has been quantified, and will be very useful in time-domain radar studies for ground-penetrating radar, free-space layered-material measurement systems, et'c.

\section{ACKNOWLEDGMENTS}

Thanks are due to several individuals who assisted in this experimental effort. Joseph Lundstrom helped modify the TEM-hom antennas for best EM performance (here at Sandia). Kwang Min (at Wright Lab) has been a primary supporter of this research, and Marcelious Willis (at Wright Lab) assisted in the measurement effort there.

\section{DISCLAIMER}

This report was prepared as an account of work sponsored by an agency of the United States Government. Neither the United States Government nor any agency thereof, nor any of their employees, makes any warranty, express or implied, or assumes any legal liability or responsibility for the accuracy, completeness, or usefulness of any information, apparatus, product, or process disclosed, or represents that its use would not infringe privately owned rights. Reference herein to any specific commercial product, process, or service by trade name, trademark, manufacturer, or otherwise does not necessarily constitute or imply its endorsement, recommendation, or favoring by the United States Government or any agency thereof. The views and opinions of authors expressed herein do not necessarily state or reflect those of the United States Government or any agency thereof. 